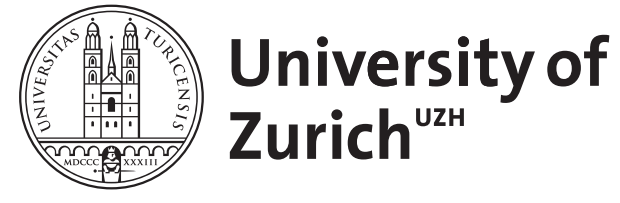

Zurich Open Repository and Archive

University of Zurich

University Library

Strickhofstrasse 39

CH-8057 Zurich

www.zora.uzh.ch

Year: 1990

\title{
Transcutane partialdruckmessung
}

Buch, Renate

DOI: https://doi.org/10.1515/bmte.1990.35.s1.24

Posted at the Zurich Open Repository and Archive, University of Zurich

ZORA URL: https://doi.org/10.5167/uzh-155434

Journal Article

Published Version

Originally published at:

Buch, Renate (1990). Transcutane partialdruckmessung. Biomedizinische Technik. Biomedical engineering, 35(s1):24-26.

DOI: https://doi.org/10.1515/bmte.1990.35.s1.24 


\title{
TRANSCUTANE PARTIALDRUCKMESSUNG
}

\author{
Renate Huch
}

Klinik für Geburtshilfe, Departement für Frauenheilkunde, Universität Zürich

Baumberger und Goodfriend berichteten 1951 über erste Versuche, unblutig die arteriellen Blutgase zu bestimmen. Mit einer Quecksilbertropfelektrode wurde der Po2 gemessen, der sich in einer erwärmten Flüssigkeitsschicht einstellte, die in engem Kontakt mit der Haut war. Rooth und Mitarbeiter wiederholten 1967 mit einem ähnlichen Messaufbau mit der dann verfügbaren Clark-Po2-Elektrode diesen Versuch. Nach ausreichender Zeit der Aequilibrierung konnte in einer $45^{\circ} \mathrm{C}$ warmen Flüssigkeit, die einen Finger umgab, ein $\mathrm{Po}_{2}$ gemessen werden, der grössenordnungsmässig dem arteriellen $\mathrm{Po}_{2}$ entsprach. Systematische und kontinuierliche HautoberflächenPo2-Messungen wurden dann 1966-67 von Evans und Naylor durchgeführt. Ihre Po2-Elektrodenentwicklung wurde mit einer Federwaage relativ lastfrei auf der Haut positioniert. Mit dieser experimentellen Anordnung konnte gezeigt werden, dass Blutgasmessungen durch die intakte Haut (=transcutan) prinzipiell biologisch und technisch möglich sind. Ihre und die Untersuchungen von Lübbers (1966) lieferten die Basis für die klinisch einsetzbaren Elektrodenentwicklungen, die zunächst für die Sauerstoffpartialdruckmessung verwirklicht wurden (Eberhardt, P., Hammacher, K., Mindt, W., 1972; Huch, A., Huch, R., Meinzer, K. und Lübbers, D.W., 1972). Als entscheidende Voraussetzung für die Messung vorı Blutgaswerten mit enger Korrelation zu den arteriellen Partialdrucken stellte sich die ausreichende Hyperämisierung des Hautareals heraus, die durch die Beheizung der Elektrode selbst erreicht wurde.

Setzt man eine unbeheizte Po2-Elektrode auf die Haut, so misst man einen $\mathrm{PO}_{2}$ in der Grössenordnung von wenigen bis null $\mathrm{mm}$ $\mathrm{Hg}$ (unter $1 \mathrm{kPa}$ ). Offenbar ist die lokale Gewebeversorgung so geregelt, dass der Gradient vom arteriellen Teil der Kapillare zur obersten Schicht der Haut auf Null abfällt. Wird die Regulation durch chemische oder physikalische Einflüsse gestört, steigt der transcutan zu messende Partialdruck für $\mathrm{O}_{2}$ an bzw. fällt $\mathrm{ab}$ für $\mathrm{CO}_{2}$. Die Voraussetzungen zur Messung von $\mathrm{Po} 2$ und $\mathrm{PcO}$ Werten auf der Haut in der Grössenordnung arterieller Werte sind um so günstiger, je besser es gelingt, die Durchblutung gegenüber der Ruhedurchblutung zu steigern. Unter diesen Bedingungen wird der $\mathrm{O}_{2}$-Verbrauch bzw. die $\mathrm{Co}_{2}$-Produktion des Hautgewebes relativ klein gegen das Angebot bzw. den Abtransport durch die Mehrdurchblutung, so dass sich die endkapillären Werte den arteriellen Drucken nähem.

Dauerhafte Hyperämie ist nach den entsprechenden Untersuchungen nur durch lokale Hyperthermie zu erzielen, entweder durch direkte Beheizung der Elektrode oder durch Diathermie. Andere Methoden zur Gefässdilatation und Durchblutungssteigerung - z.B. pharmakologische - haben sich bisher als nicht geeignet erwiesen, eine für die transcutane Messung geeignete Arterialisierung des Hautkapillargebietes zu bewirken.

\section{MESSPRINZIP TRANSCUTANER $\mathrm{PO}_{2}$ (tcPo2)}

tcPo2 wird polarographisch mit einer Clark-Typ-Elektrode bestimmt, die in den Abmessungen einer EKG-Elektrode ähnlich und mit einem doppelseitig klebenden Ring ebenfalls wie eine EKG-Elektrode auf der haarlosen Haut fixiert wird. Die Kathoden sind in der Regel aus Platin oder Gold. Je nach Durchmesser der Kathoden dienen die meist ringförmig die Kathode(n) umgebende Silberanode oder die Kathode selbst zur Aufnahme einer Heizwicklung zur Beheizung der Elektrode. Eine Elektrodenkerntemperatur zwischen $44-45^{\circ} \mathrm{C}$ ist für die Erzielung einer ausreichenden Hyperämie notwendig. Die Wahl der Clark-Membran wird in Bezug auf die Sauerstoffdurchlässigkeit und Dicke durch den $\mathrm{O}_{2}$-Eigenverbrauch der Kathode bzw. ihren Durchmesser bestimmt. Molekularer Sauerstoff diffundiert durch die gefässlose Epidermis und die 
Elektrolyt-Membranschicht der Elektrode zu der Kathode und wird dort reduziert. Der Reduktionsstrom ist dem Sauerstoffdruck proportional. Die Eichkurve ist linear und kann mit 2 Eichpunkten - am einfachsten mit Stickstoff und Luft festgelegt werden.

\section{MESSPRINZIP TRANSCUTANER PcO2 (tcPcon)}

tcPcO2 wird potentiometrisch mit miniaturisierten membranbespannten pH-Elektroden nach dem Prinzip von Stow and Randall (1954) mit Glas- oder mit Metall-MetalloxydElektroden (Hopmeier und Ullrich, 1985) gemessen. Auch die tcPCO 2 -Elektrode wird direkt beheizt; Elektrodenkerntemperaturen zwischen $42-43^{\circ} \mathrm{C}$ reichen hier bereits aus, um die Hyperämie derart zu gestalten, dass zwischen transcutanen und arteriellen Partialdruckwerten eine relativ konstante Beziehung besteht (Severinghaus, J.W., Stafford, M., Bradley, A.F., 1978). Co2 diffundiert aus den durch Hyperthermie dilatierten Kapillaren in die Natrumbicarbonat-Elektrolytlösung und verändert den $\mathrm{pH}$-Wert. Diese $\mathrm{pH}$-Aenderung ist proportional dem $\mathrm{CO}_{2}$-Partialdruck. Die Eichung der transcutanen $\mathrm{PcO}_{2}$ Elektrode erfolgt ebenfalls vor und nach jeder Hautmessung in vitro mit 2 Gasen bekannten $\mathrm{CO}_{2}$-Druckes.

Die Aufzeichnung der Heizleistung zur Konstanthaltung der vorgegebenen Elektrodenkerntemperatur in der tcPo2- und tcPco2-Elektrode kann als Mass für die relative lokale Hautperfusion genutzt werden. Fliesst mehr (kühlendes) Blut, muss die Heizleistung ansteigen; die relative lokale Hautperfusion nimmt zu.

Da in unserer Gruppe die Faktoren, die bei der transcutanen Messung der Gasdrucke Einfluss nehmen, systematischer für die Sauerstoffelektrode studiert wurden und auch die klinischen Erfahrungen grösser sind (Huch, R., Huch, A., Lübbers, D.W., 1981), sollen im folgenden die Verhältnisse am Beispiel der transcutanen $\mathrm{Po}_{2}$-Elektrode dargestellt werden.

Abb. 1 zeigt schematisch die Faktoren, die bei transcutaner Po2Messung in den Po2 erhöhender ( $\boldsymbol{\uparrow}$ ) oder erniedrigender Weise Einfluss ( $\downarrow$ ) nehmen.

Ein entscheidender, wenn nicht der entscheidende Faktor, um unabhăngig von Schwankungen der lokalen Hautperfusion Po2Werte mit konstantem Bezug zu arteriellen Partialdruckwerten messen zu können, ist das Ausmass der Hyperămie, das, wie geschildert, offenbar nur durch Hyperthermie erreicht werden kann. Die Elektrodenkemtemperatur, die nicht identisch ist mit der resultierenden Hautoberflachentemperatur, sondern ca. $2^{\circ} \mathrm{C}$ höher ist, muss fur eine ausreichende Hyperamie zwischen $44^{\circ} \mathrm{C}$ (bei der Messung beim Feten in utero und beim Fruhgeborenen)

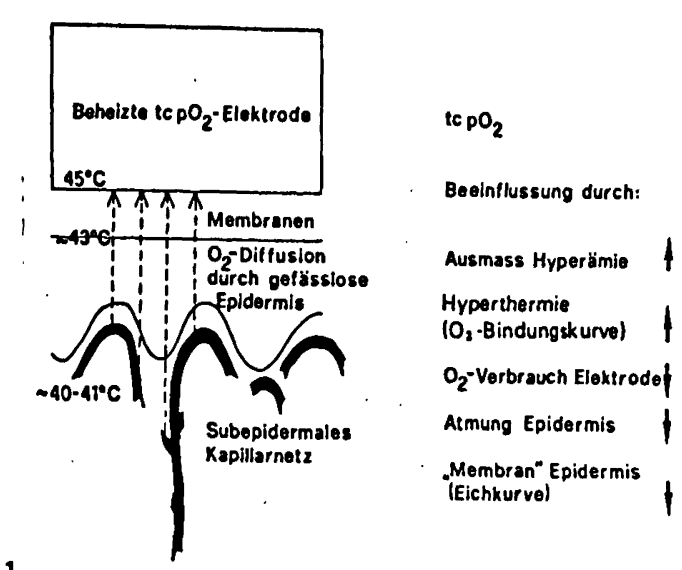

Abb. 1

und $45^{\circ} \mathrm{C}$ (bei der Messung beim Neugeborenen oder Erwachsenen) liegen. In Abhängigkeit von der Dauer der Fixierung der Elektrode und der peripheren Durchblutung, die in Präschock- oder Schocksituationen gestört sein kann, steigt bei diesen Temperaturen das Risiko einer Hautläsion. Die relativ hohen Temperaturen sind aber für die korrekte Erfassung der arteriellen Werte unerlässlich. Dies ist mit stufenweiser Steigerung der Elektrodenkerntemperatur bei Luft- und Sauerstoffatmung bzw. stufenweiser Drosselung der Hautdurchblutung eindrücklich zu belegen.

Ein zweiter wichtiger Einfluss ist der Effekt der Hyperthermie auf die Lage der Hämoglobinbindungskurve, die bekanntlich temperaturabhängig ist. Bei konstantem $\mathrm{O}_{2}$ - bzw. $\mathrm{Co}_{2}$-Gehalt steigen mit jedem Grad Celsius der $\mathrm{P}_{2}$ und der $\mathrm{Pco}_{2}$ um rund 5\% an (Bradley, H.F., Stupfel, M., Severinghaus, J.W., 1956; Gleichmann, U. und Lübbers, D.W., 1960). Das Ausmass der "künstlichen" $\mathrm{Po}_{2}$ - und $\mathrm{PcO}_{2}$-Erhöhung gegenüber den arteriellen Werten wird von dem Temperaturgradienten zwischen Elektrodenkern und Kapillar-gebiet beeinflusst, der seinerseits stark von der jeweiligen Epidermisstärke an der Messstelle abhängig ist. Bei Früh- und Neugeborenen ist daher der erhöhende Effekt der Temperatur besonders gross. Er kann dazu führen, dass die transcutanen Po2-Werte höher als die arteriellen sind.

Nicht zu vernachlässigen ist der Eigenverbrauch der Sauerstoffelektrode (ein Faktor, der bei der Pco2-Elektrode entfallt). Jede Sauerstoffelektrode entnimmt dem Messmedium Sauerstoff und verändert damit die zu messende Grösse. Da der $\mathrm{O}_{2}$-Eigenverbrauch der Kathode etwa quadratisch mit der Kathoden-Oberfläche wächst, kann auf diese Störgrösse durch die Verwendung sehr kleiner Kathoden Einfluss genommen werden. Bei Verwendung grosser Kathoden muss eine Kompensation mit Membranen mit grosser Schichtdicke oder geringer Sauerstoffpermeabilitat erfolgen. Die in unseren Messsystemen verwandien $15 \mu \mathrm{m}$ Platinkathoden haben einen $\mathrm{O}_{2}$-Eigenverbrauch entsprechend einem Abfall von $0.015 \mathrm{~mm}$ $\mathrm{H}_{\mathrm{g}}$ pro Minute bei einem $\mathrm{PO}_{2}$ von $100 \mathrm{mmll}$. 
Wenig zu beeinflussen ist der Effekt der Epidermis-Atmung, die durch die Hyperthermie gegenuber der normalen Hautatmung sogar gesteigert ist. Nach unseren Untersuchungen ist der Effekt insgesamt jedoch relativ gering, in der Grossenordnung um 5 $\mathrm{mm} \mathrm{Hg}$ bei Luftatmung.

Individuell ein grösseres Ausmass kann der zuletzt in Abb. 1 aufgefuhrte Faktor "Membran" Haut haben. Grob vereinfacht kann die gefassslose Epidermis bei der in vitro-Eichung wie eine zweite Clark-Membran betrachtet werden, die nicht mitzuerfassen ist. Diese sog. zweite Clark-Membran verändert die in vitro bestimmte Einstellzeit der Elektrode und erniedrigt das Mess-Signal.

\section{KLINISCHE ERFAHRUNGEN UND BEDEUTUNG DER} BLUTGASMESSUNG BEI DER UEBERWACHUNG VON PATIENTEN

In zahlreichen klinischen Studien konnte gezeigt werden, dass die transcutanen Po2-Werte, die auf der Basis einer einfachen in vitro-Eichung ermittelt werden, verlässlich die arteriellen Werte reflektieren. Die oben dargestellten Faktoren kompensieren sich, oft in idealer Weise, so dass tcPo2 und PaO2 eine 1:1 Relation erhalten.

Früh- und Neugeborene haben sich als ideale Population für die Anwendung der Methodik erwiesen. Erst schwer pathologische Kreislaufsituationen, die in der Regel klinisch erkennbar sind, führen durch die Kreislaufzentralisation zur fehlerhaften Unterschätzung. der arteriellen Werte. Beim kranken Erwachsenen hat die Anwendung der Technik in dieser Hinsicht häufiger Limitierungen. Mit Ausnahme schwerer Schockzustände ist die Differenzierung zwischen Hautperfusions- oder Atmungs-bedingten tcPon-Abfällen (oder Pco2-Anstiegen) oft nicht möglich; starke Blutdruckabhängigkeiten wurden beim Kranken beobachtet (Huch, R., Huch, A., Lübbers, D.W., 1981). Hingegen gibt es gute Erfahrungen bei physiologischen Studien oder generell in Fällen stabiler Kreislaufverhältnisse. Erwähnt sei die Anwendung in der Sport-, Höhen- und Tauchmedizin, bei Lungenfunktionsmessungen, bei Schlafstudien, pharmakologischen Untersuchungen u.v.m.

Seit der Verfügbarkeit der Pulsoximetrie zur Messung der Sauerstoffsättigung, die im Gegensatz zur transcutanen Partialdruckmessung keine Vorbereitungs- und Eichprozeduren erfordert und nach den vorliegenden Erfahrungen beim Erwachsenen relativ zuverlässig funktioniert, stellt sich die Frage, wo künftig die transcutane Partialdruckmessung ihren Einsatzbereich hat. Generell wird sie unverzichtar sein, wenn Veränderungen im oberen flachen Teil der Hämoglobinbin- dungskurve oder ausserhalb, also bei Hyperoxămie, liberwacht werden sollen. Dies wie auch die geringere Anfalligkeit gegen bewegungsbedingte Artefakte răumen der Methodik einen wichtigen Platz bei der Neugeborenenüberwachung ein. Das kürzlich publizierte Statement der American Academy of Pediatrics (1989) zum Stellenwert der Methodik in der neonatalen Intensivüberwachung hat allerdings erneut deutlich gemacht, dass die Zuverlăssigkeit der Methode von der korrekten Anwendung und vom Verständnis der physiologischtechnischen Besonderheiten der transcutanen Partialdruckmessung entscheidend abhängig ist.

\section{Referenzen:}

American Academy of Pediatrics. Report of Consensus Meeting December 1986: Pediatrics 83: 122, 1989

Baumberger JP, Goodfriend RB: Determination of arterial oxygen tension in man by equilibration through intact skin. Fed Proc 10: 10, 1951

Bradley AF, Stupfel M, Severinghaus JW: Effect of temperature on PcO2 and Poz of blood in vitro. J Appl Physiol 9: 201, 1956

Eberhard P, Hammacher K, Mindt W: Perkutane Messung des Sauerstoffpartialdruckes. Methodik und Anwendungen. Proc. "Medizin-Technik 1972", Stuttgart: 26, 1972

Evans NTS, Naylor PFD: The dynamics of changes in dermal oxygen tension. Respir Physiol 2: 61, 1966/67

Evans NTS, Naylor PFD: The systemic oxygen supply to the surface of human skin. Respir Physiol 3: 21, 1967

Evans NTS, Naylor PFD: The oxygen tension gradient across human epidermis. Respir Physiol 3: 38, 1967

Gleichmann U, Lübbers DW: Die Messung des Sauerstoffdruckes in Gasen und Flüssigkeiten mit der PtElektrode unter besonderer Berücksichtigung der Messung im Blut. Pflügers Arch ges Physiol 271: 431, 1960

Hopmeier J, Ullrich GJ: Die Transcapnode :- ein Messwertaufnehmer zur transcutanen $\mathrm{Pco}_{2}$ Messung mit einem Iridium-pH-Sensor. Biomed Technik 30: 94, 1985

Huch A, Huch R, Meinzer K, Lübbers DW: Eine schnelle, beheizte Pt-Oberflächenelektrode zur kontinuierlichen Ueberwachung des Pon beim Menschen. Elektrodenaufbau- und eigenschaften. Proc. "Medizin-Technik 1972". Stuttgart: 26, 1972

Huch R, Huch A, Lübbers DW: Transcutaneous Po2. Thieme, Stuttgart: 1, 1981

Lübbers DW: Methods of measuring oxygen tensions of blood and organ surfaces. In: Oxygen measurements in blood and tissues, ed by Payne JP, Hill DW. J \& A Churchill, London: 103,1966

Rooth G, Sjöstedt S, Caligara F: Bloodless determination of arterial oxygen tension by polarography. Sci Tools, The LKW Instrument J 4: 37, 1957

Severinghaus JW, Stafford M, Bradley AF: tcPo2 electrode design, calibration and temperature gradient problems. Acta Anaesthesiol Scand Suppl 68: 118, 1978

Stow RW, Randall BF: Electrical measurement of the Pcon blood. Amer J Physiol 179: 678, 1954 